\title{
Continuously Rotating Energy Harvester with Maximum Power Point Tracking
}

\author{
Tzern T. Toh, Paul D. Mitcheson, Andrew S. Holmes and Eric M.Yeatman \\ Department of Electrical and Electronic Engineering, Imperial College London, UK. \\ E-mail: tzern.toh@imperial.ac.uk and e.yeatman@imperial.ac.uk
}

\begin{abstract}
In this paper, we analyse and demonstrate an energy harvesting generator powered by continuous rotation, using gravitational torque to enable single point attachment. The electro-mechanical behaviour of the generator is presented, alongside experimental results from an implementation based on a conventional DC motor. The off-axis performance is also modelled. The design and experimental results for an adaptive power processing circuit are also presented, as the first demonstration of maximum power point tracking for a motion energy harvester.
\end{abstract}

\section{Keywords: Energy scavenging, machine monitoring, power convertor}

\section{Introduction}

Energy harvesting from moving structures has been a topic of much research, particularly for applications in powering wireless sensors [1]. Most motion energy harvesters are inertial, drawing power from the relative motion between an oscillating proof mass and the frame from which it is suspended [2]. The inertia of the proof mass provides a counter-force to that presented by the source of motion, so that no second point of attachment is needed - this greatly expands the possible range of installations and applications, particularly for highly miniature devices. While most reported devices use linear relative motion between the mass and frame, rotating motion is also possible [3]; in fact, rotating mass inertial generators have been the more commercially successful to date, being well suited to the specific application of wristwatches [4]. By the use of a proof mass with a centre offset from the axis of rotation, these rotational devices can be driven by either linear or rotating source motion. However, since the inertial counter-force results from the acceleration of the frame, this effect cannot be used to extract power where the host motion is constant (whether linear or rotational).

For body motion powered devices such as wristwatch generators, constant rotation is in any case not available. However, for many important applications, including tire pressure sensing and condition monitoring of machinery, the host structure does undergo continuous rotation. In these cases, previous energy harvesters have typically been driven by the associated vibration. Here, we show that rotational motion can be used directly to harvest power and that conventional rotating machines can be easily adapted to this purpose.

A generic rotating generator consists of two main components, a stator and rotor, which are rotated with respect to each other by some external source of motion, and a transduction mechanism (generally electromagnetic) which impedes this relative motion, and by doing so converts mechanical into electrical power. An energy harvester differs from a conventional generator firstly in that it should normally apply a mechanical load small enough not to substantially impede the motion source; and secondly that it will typically be desirable to attach the harvester to the motion source at a single point, without the need to anchor it to a non-moving structure. The latter requirement creates a need for a mechanism to oppose the source rotation.

This mechanism can be straightforwardly provided by gravity, with the limitation that the axis of rotation (or a significant component of it) is horizontal. To create gravitational torque, the generator part not attached to the host should have a centre of mass offset from the axis of rotation. Two possible variants are illustrated in figure 1. 


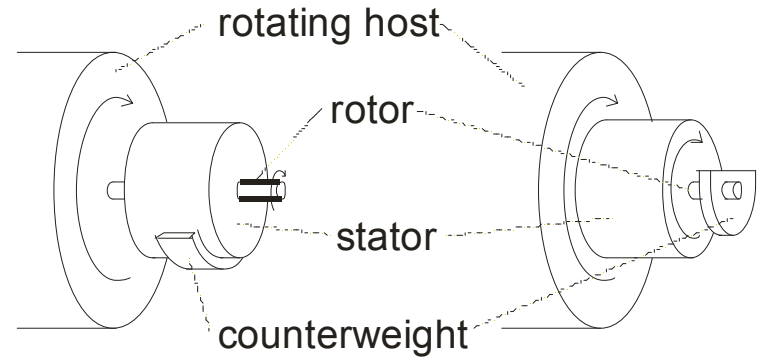

(a)

(b)

Figure 1. Possible implementations of the gravitational torque rotating energy harvester: (a) rotation of the rotor, with an offset mass on the stator; and (b) rotation of the stator with an offset mass on the rotor.

Here the stator and rotor are so labelled according to their behaviour (static or rotating) in a conventional generator implementation, so that the rotor is the inner module which includes the axle, and the stator is the outer housing and associated structures. Either can be attached to the rotating host, with an offset mass attached to the other. The force of gravity on this offset mass tends to keep it oriented downwards, thus impeding the rotation of that module, while the other module is forced to rotate along with the host. This creates the possibility of generating power.

\section{Rotational generator analysis}

The gravitational torque generator can be analyzed with the aid of figure 2 . We assume that a conventional DC machine is employed, with the rotor coupled to a continuously rotating source, and an additional mass $m$ attached to the otherwise axially symmetric stator at a distance $L$ from the axis. If power is drawn, by connecting a finite load to the generator output terminals and drawing a rotor current, magnetic torque will initially rotate the stator along with the rotor and host; and this will create a gravitational torque, $T_{g}=m g L \sin (\theta)$. The position of the mass should stabilize at the angle for which $T_{g}$ equals the magnetic torque, $T_{m}$. Stable generation is thus limited to magnetic torques up to $m g L$, above which the rotor flips over. Therefore the maximum power is:

$$
P_{\max }=m g L \omega
$$

with $\omega$ the angular rotation rate of the host.

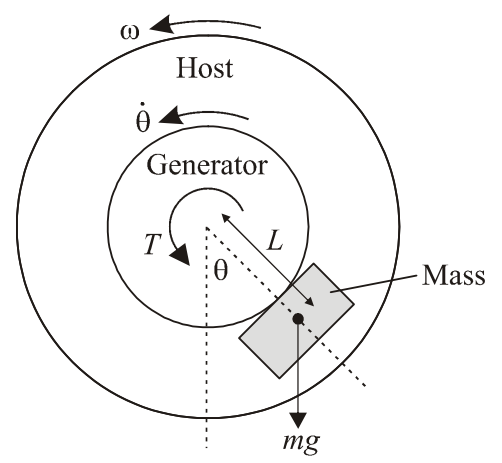

Figure 2. Schematic of the gravitational torque harvester.

For a conventional DC machine driven as a generator at a given speed, the maximum load power is achieved for a load matched to the armature resistance, giving an output electrical power of

$$
P_{\text {elec }}=\frac{\left(K_{E} \omega\right)^{2}}{4 R_{L}}
$$

with $K_{E}$ the motor constant, $R_{L}$ the load resistance, and $K_{E} \omega$ the generated electro-motive force. From this, and noting that the total electrical power is twice the load power given by (2), we can derive the maximum rotation speed before the motor flips over, $\omega_{c}$, as

$$
\omega_{c}=\frac{2 R_{L} m g L}{K_{E}^{2}}
$$

The device will continue to generate above this speed, but the motion will now be highly nonlinear, and the power levels are likely to be greatly reduced.

\section{Experimental results}

Figure 3 depicts our experimental setup, where two DC motors were coupled together at their shafts. One motor acts as a rotational source while clamped onto the workbench, and the other as the gravitational torque generator, suspended in air. On the generator's stator, we attached a rectangular mass, while a load resistor, $R_{L}$, was connected to its output terminals. We measured the angular velocity of the shaft using an optical tachometer, and the voltage across $R_{L}$ to derive the output power. In this work, $K_{E}=2.6 \times 10^{-3} \mathrm{~V} \cdot \mathrm{s} / \mathrm{rad}$, and the additional mass was $20 \mathrm{~g}$, centered $2 \mathrm{~cm}$ from the generator's shaft. 


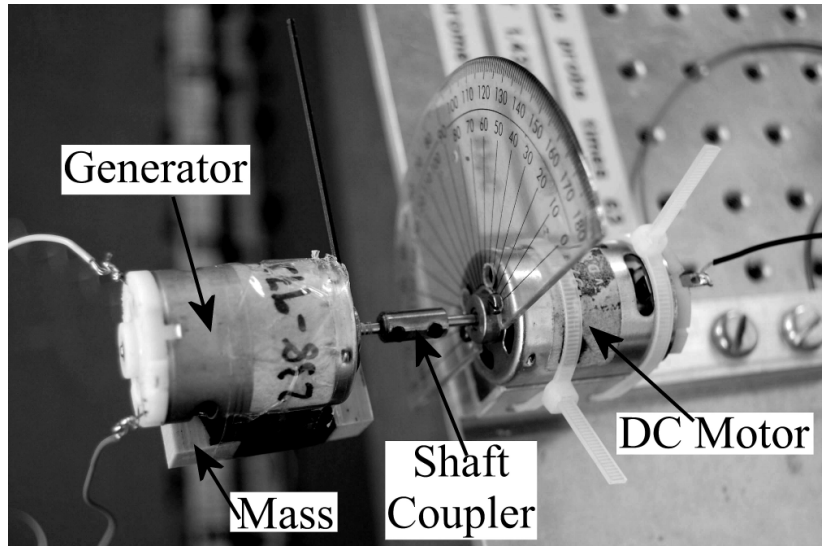

Figure 3. Experimental setup of the generator and rotational source.

Figure 4 shows the output power measured for this setup, for various values of load resistance. The highest power is obtained when $R_{L}$ is matched to the armature resistance of $1.1 \Omega$, as expected. Output power varies as rotation rate squared, in agreement with (2).

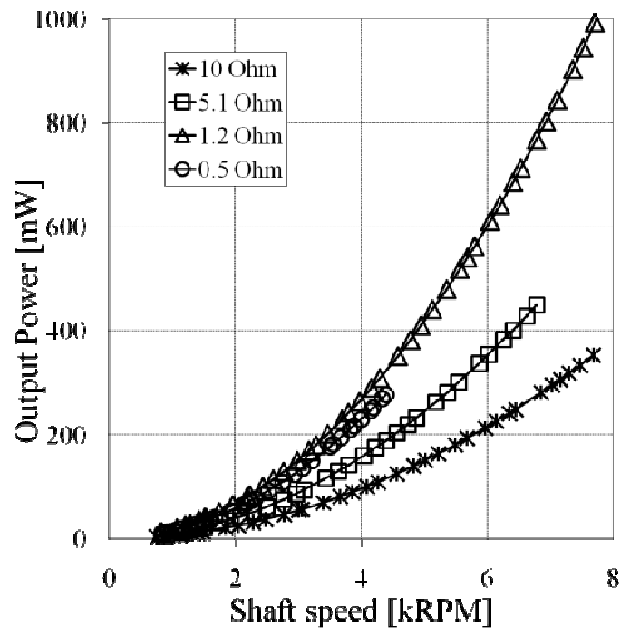

Figure 4. Measured powers for the specified load resistances when $R_{A}=1.1 \Omega$.

When $R_{L}$ is much less than $R_{A}$, the stator flips at a lower speed, since $I_{A}$ more quickly approaches a value at which the generator will produce excessive torque. In general, for on-axis operation (generator centred on host axis), we can prevent flip-over by limiting the armature current. Since the motor torque is given by $T=K_{E} I_{A}$, the current should be limited to:

$$
I_{\max }=\frac{m g L}{K_{E}}
$$

Figure 5 shows the measured and modelled flip-over speeds as a function of load resistance. Flip-over speed is seen to increase with load resistance, as a result of decreased armature current and the associated reduction in motor torque. The simple model is obtained by modifying equation (3) to the case where the load $R_{L}$ is not matched to the armature resistance $R_{A}$, giving:

$$
\omega_{c}=\frac{\left(R_{L}+R_{A}\right) m g L}{K_{E}^{2}}
$$

It can be seen that this formulation significantly overestimates $\omega_{c}$. A more accurate model is obtained by including the torque due to mechanical drag between the rotor and stator. This was approximated by fitting a function to the measured angle, $\theta$ vs. frequency for the open circuit case (no motor torque), and the result is also plotted in figure 5. Although the fit to experiments is closer, in this case $\omega_{c}$ is under-estimated; this may be as a result of neglecting contact resistance between the brushes and the commutator, which can be speed dependent.

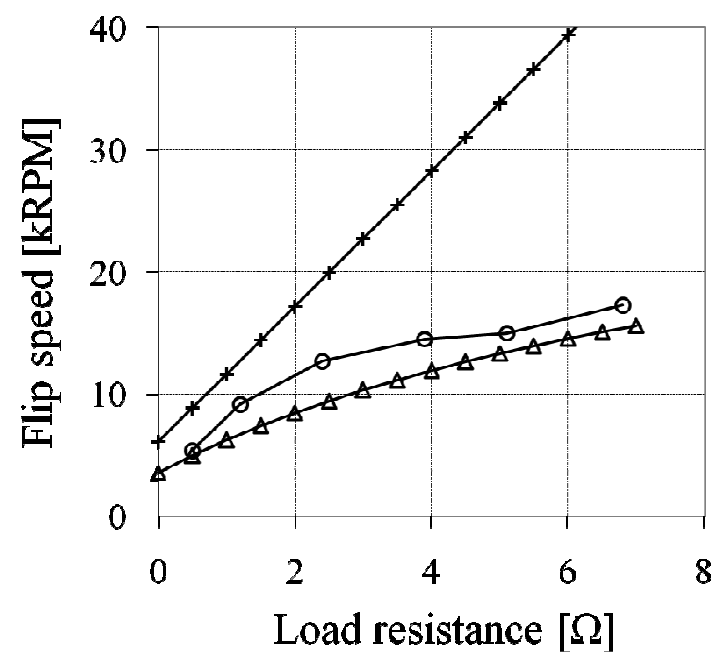

Figure 5. Flip-over speed vs. load resistance when $R_{A}=$ $1.1 \Omega$ : experimental (circles); linear model (crosses), and model including drag (triangles).

\section{Maximum power point tracking}

From the Section 3, optimal power transfer from the generator to the load was achieved when $R_{L} \approx R_{A}$. Given the relatively small value of $R_{A}$ compared to the input impedance of a device powered by this generator, a Boost Switch Mode Power Supply (SMPS) was 
constructed to implement this impedance match as well as to provide a smooth input current [5]. The input impedance, $R_{I N}$ of a Boost SMPS can be altered to be less than the load impedance, $R_{L}$, by varying the duty cycle, $\delta$ :

$$
R_{I N}=R_{L}(1-\delta)^{2}
$$

A control loop measures the input voltage of the SMPS. As the required input impedance of the converter is known and equal to $R_{A}$, this measurement of input voltage allows the generation of a current demand reference signal. The existing input current is measured using a sense resistor at the Boost SMPS input which is then amplified and low pass filtered to remove the effect of current ripple due to the switching cycle of the power converter. This measure is then compared with the current demand reference, and the error is reduced using a proportional and integral (PI) controller. From the PI-controller, a pulse-width modulated (PWM) signal is generated with the appropriate duty cycle, which is used to drive the switching transistor in the Boost SMPS.

Figure 6 shows the block diagram of the circuitry used to implement and test this adaptive maximum power point tracking. A bench power supply was used to mimic the rotational generator's EMF, and $R_{A}$ was represented by a series connected resistor. The PI control loop was realized using a PIC18F452 microprocessor [6].

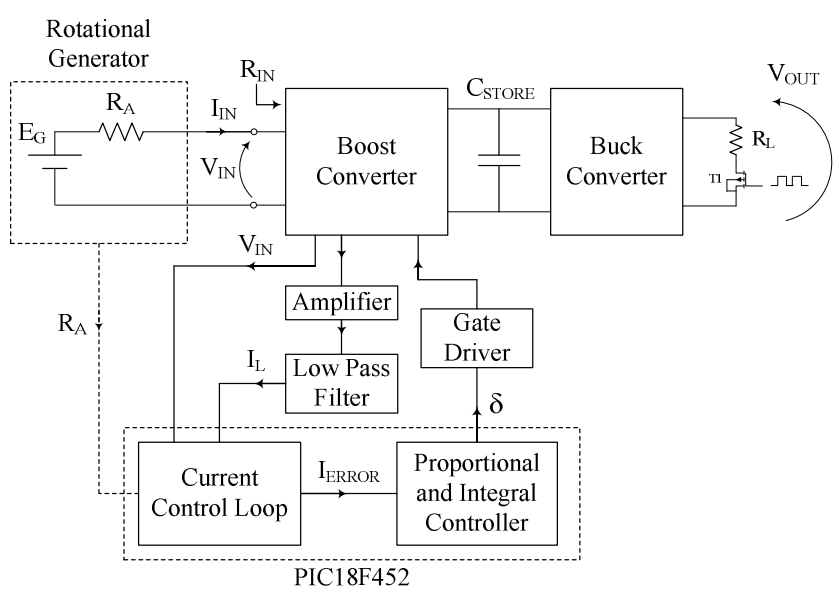

Figure 6. Schematic of the power processing circuitry.

The gains of the PI-controller were tuned by inspection and their values were chosen based on the amount of jitter present in the PWM gate drive signal and response time of the controller. A $100 \mathrm{kHz}$ PWM signal generated from the PIC was passed through a gate driver integrated circuit to reduce the Miller plateau present in the Boost's switching transistor in order to reduce converter switching losses.

A capacitor $C_{S T O R E}$ charges when excess power is being generated (i.e. when the generator is producing more power than is being consumed by the load), and $C_{\text {STORE }}$ discharges when there is not enough power being generated to feed the load. It is essential that the supplied output voltage is regulated when it is used to power a device. Output voltage regulation is performed by an off-the-shelf wide input, regulated Buck SMPS [7].

Transistor $T 1$ was placed in series with $R_{L}$ to simulate variations in load, and also to demonstrate the capabilities of $C_{\text {STORE }}$ to continuously provide sufficient power to $R_{L}$. The gate of $T 1$ was switched at a rate of $0.3 \mathrm{~Hz}$ to simulate these occurrences. With $T 1$ on, the Buck SMPS will have to draw more input current to regulate the output voltage and supply the required load current. When $T 1$ is off, minimal current is drawn by the Buck converter and hence, $C_{\text {STORE }}$ will increase its stored energy if the generator is still generating.

Characterisation of the Boost SMPS was done by applying an input voltage of $5 \mathrm{~V}$ and using a load resistor of $1 \mathrm{k} \Omega$. A maximum voltage gain of approximately 5.2 was obtained at a duty cycle of $93 \%$ before the voltage gain rolls off due to parasitic losses in the circuit. This is shown in figure 7. Consequently, we have limited the duty cycle generated by the PIC to $93 \%$ in order to maintain a monotonic response between duty cycle and voltage gain.

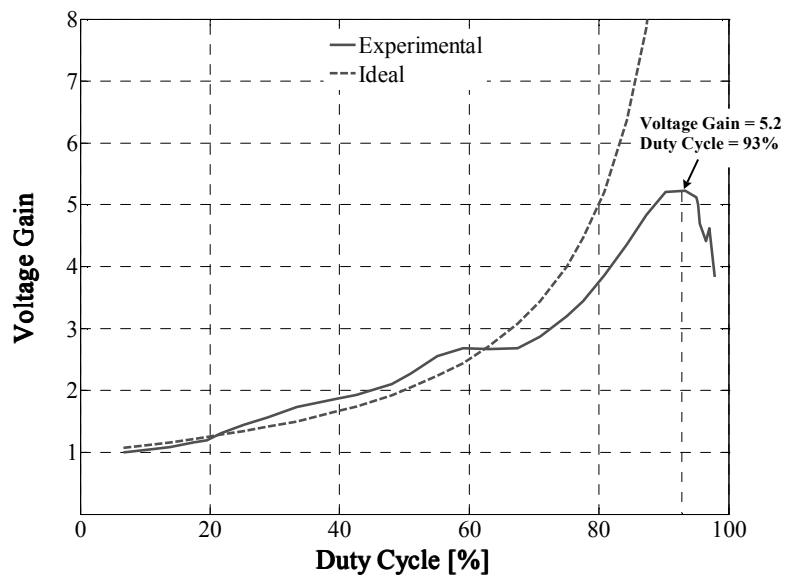

Figure 7. Voltage gain characteristics of the Boost SMPS.

To determine how well the Boost converter performs impedance matching, $R_{A}$ (and therefore the converter input impedance) was set to a test value of $9.1 \Omega$ and $R_{L}$ 
values of $50 \Omega$ and $100 \Omega$ were connected across $C_{\text {STORE }}$ $(23 \mathrm{mF})$ as $V_{I N}$ was changed. An $R_{A}$ of $9.1 \Omega$ was chosen because the circuit has been designed to be used with a different DC motor to that used in the original experimental work. For this experiment, the Buck converter was disconnected. Figure 8 shows a proportional variation in input current to changes in input voltages, justifying a constant input impedance of approximately $9.1 \Omega$ for different load resistances.

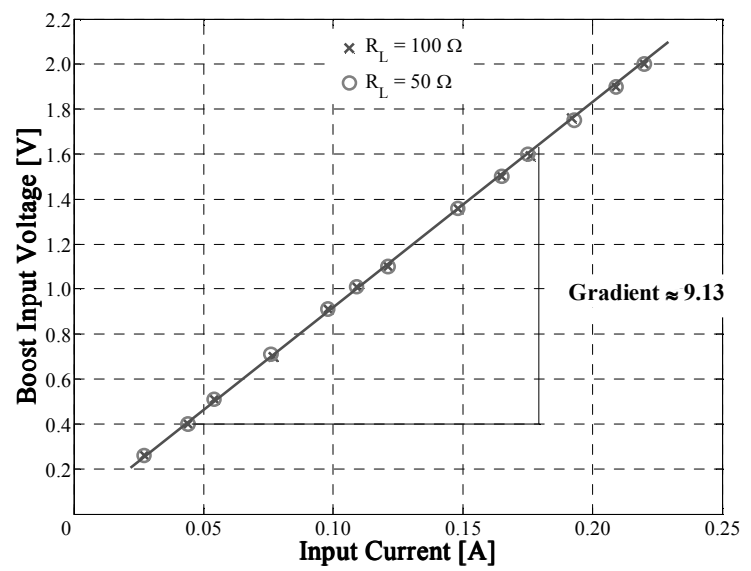

Figure 8. Impedance matching performance of the current controller.

Figure 9 illustrates the changes in duty cycle and correspondingly, the voltage across the storage capacitor, while the input impedance of the Boost SMPS was continuously matched to $R_{A}$. Again, the buck converter was removed and the load resistance was connected directly to the output of the boost converter. The deviation in input current when two different loads were used was barely noticeable.
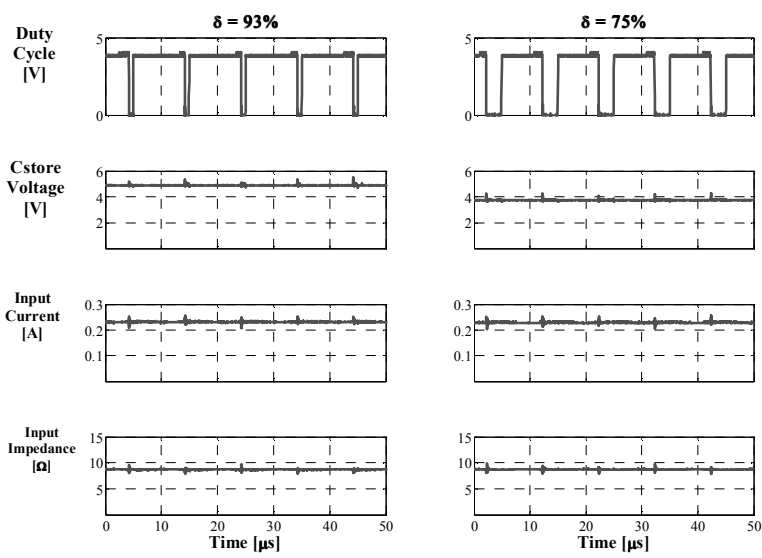

Figure 9. Variations in duty cycle under different loads to achieve an input impedance of $9.1 \Omega$.
The graphs in figure 10 show the waveforms from the complete circuit (including output regulation with the Buck converter) with $R_{A}=9.1 \Omega$ and $R_{L}=27 \Omega$. When Tl's gate is driven high, it turns on causing a load current to be drawn. This results in a discharge of the storage capacitor, because the Buck SMPS requires more input power to regulate the output voltage at $3.3 \mathrm{~V}$ than is being generated. When $T 1$ is off, $C_{\text {STORE }}$ increases its charge, as expected.

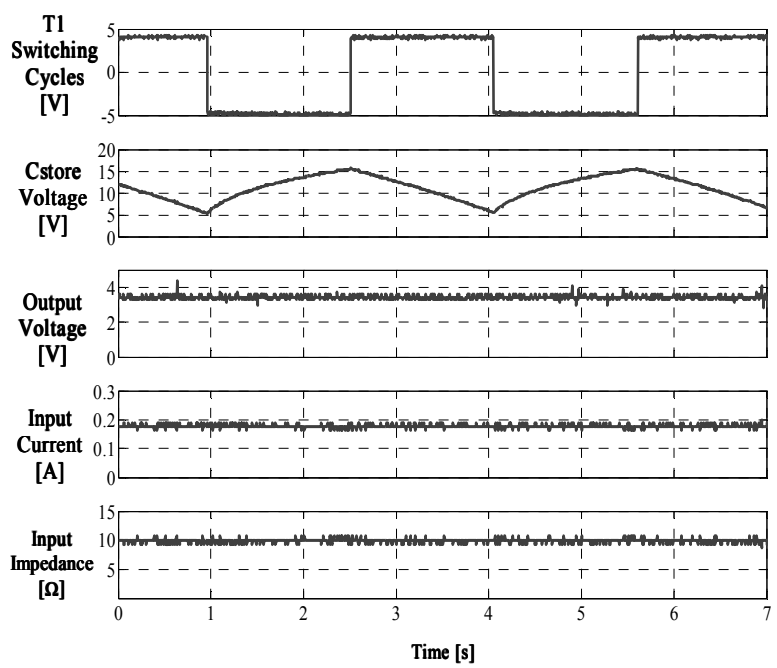

Figure 10. The role of $C_{\text {STORE }}$ in regulating the output voltage during high and low loads, while keeping the input impedance matched to $9.1 \Omega$.

Throughout the on and off intervals of $T 1$, the input current remains constant and hence, the input impedance of the Boost SMPS was matched to $R_{A}$. Therefore, maximum power can be transferred from the rotational generator to the load. When excess power is generated, the excess energy is stored in $C_{\text {STORE}}$, so that the output voltage can still be regulated when too little power is being generated.

There will be situations where the rotational generator's speed will vary, and we have simulated this condition by altering the Boost's input voltage by adjusting the bench power supply. Also, T1 was removed, leaving $R_{L}$ connected directly across the Buck SMPS output. It was observed that the input current changes proportionally to the variations in voltage, as required. As the generator's speed increases, more power is generated than is consumed by the load, leading to an increase in the voltage across $C_{S T O R E}$. Subsequently, when the speed decreases, $C_{\text {STORE }}$ will discharge to provide the necessary power to the Buck SMPS. 
From the plots in figure 11, it can be seen that the output voltage stays regulated at $3.3 \mathrm{~V}$ and, as importantly, the input impedance stays matched to $R_{A}-$ an essential condition for harvesting energy optimally from a rotational source in practical situations.

It should be noted that the end-to-end efficiency of the power processing circuit has not yet been evaluated, or optimized. However, this circuit does serve as a proofof-concept for, and first demonstration of, maximum power point tracking for a motion energy harvesting generator.

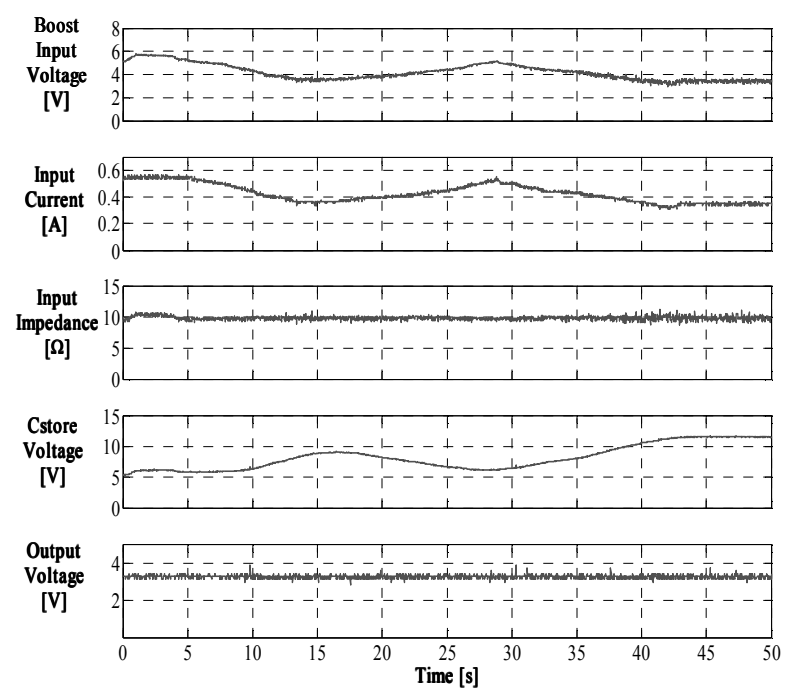

Figure 11. Performance of the matching circuit for a varying input voltage and fixed load, showing that the input impedance remains matched to $R_{A}$.

\section{Off-axis operation}

Whilst there are applications in which this energy harvester could be used with its centre of rotation aligned with the centre of rotation of the host, it will often be useful, and in some instances essential, to attach the harvester off-axis. This is illustrated in figure 12.

If the centres of rotation are misaligned (increasing $l_{1}$ ), the entire generator is subjected to a centripetal force. This has the effect of causing the offset mass, $m$, to be thrown outwards and thus the rate of change of $\left(\theta_{1}-\theta_{2}\right)$ reduces. As it is this rate of change that causes power to be generated, the offset position of the generator will tend to reduce the power generated.

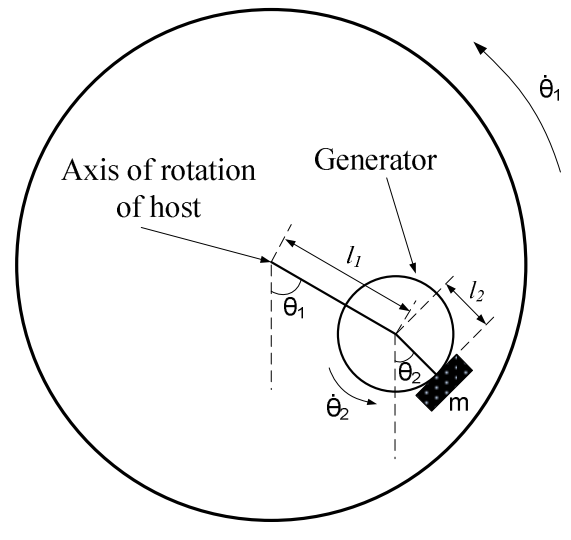

Figure 12. Schematic of the off-axis generator.

As shown in figure 12, this mechanical problem is essentially that of the double pendulum, where the first link is driven at constant rotational speed, i.e. $\dot{\theta}_{1}$ is constant. The equation of motion describing the rotation of the mass, $m$, through the angle $\theta_{2}$ can be derived as:

$$
\begin{aligned}
\ddot{\theta}_{2}= & \frac{l_{1} \dot{\theta}_{1}^{2} \sin \left(\theta_{1}-\theta_{2}\right)-l_{1} \ddot{\theta}_{1} \cos \left(\theta_{1}-\theta_{2}\right)-g \sin \left(\theta_{2}\right)}{l_{2}} \\
& -\frac{K_{E}^{2}\left(\dot{\theta}_{2}-\dot{\theta}_{1}\right)}{2 m l_{2}^{2} R_{A}}
\end{aligned}
$$

This is the well-known equation of motion for a double pendulum, with an additional term representing the damping due to the generator as described in section 2. Analytical solutions to the motion of the double pendulum system are not possible even when $\dot{\theta}_{1}$ is constant, as the system is non-linear and chaotic, and so in order to determine the importance of this centripetal effect on power generation, a Simulink model of a double pendulum system was built to investigate the behaviour numerically. This model implements equation (7), with the values of the physical parameters being taken from those of the generator used in the experimental work, assuming a matched load resistance. The host speed is linearly increased from zero to 50 rad. $\mathrm{s}^{-1}$ (about $500 \mathrm{rpm}$ ).

With no non-linear effect (i.e. $l_{l}=0$ ), the system is expected to generate power proportional to the square of rotational angular velocity. This behaviour is confirmed by simulation, and is illustrated by the smooth line in figure 13. Figure 13 also shows the instantaneous power (after moderate low pass filtering to reduce the most rapid oscillations), for two non-zero values of offset distance. The host speed is linearly increased from zero to $50 \mathrm{rad} . \mathrm{s}^{-1}$. As can be seen, the 
power initially rises as in the on-axis case, but with oscillations of increasing magnitude. For the larger offset, the centripetal acceleration eventually overwhelms the gravitational effect, and the power begins to drop.

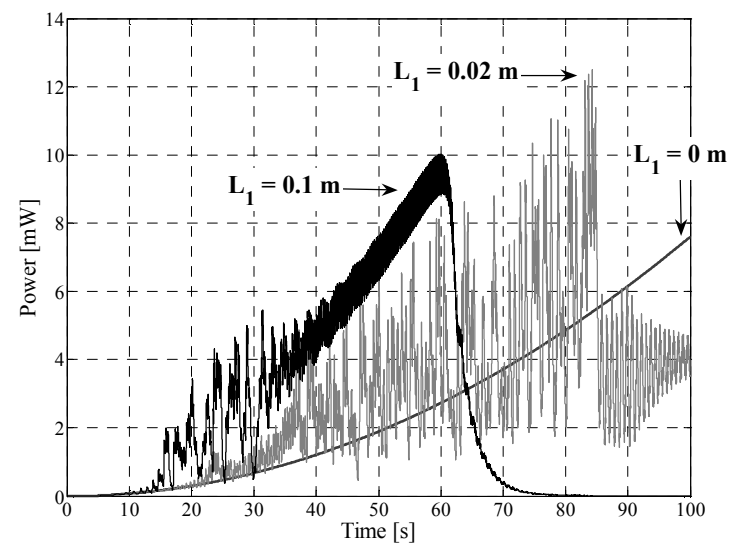

Figure 13. Generated power vs. time as host rotation rate is ramped linearly from 0 to $50 \mathrm{rad}^{-1} \mathrm{~s}^{-1}$, for offset distances $l_{l}$ as indicated.

A much lower speed range was chosen for figure 13 than for the experimental (on-axis) values of figure 4, because of the low flip-over speeds at higher offsets. However, the on-axis simulation in figure $13\left(\mathrm{~L}_{1}=0\right)$ agrees well with the matched load line of figure 4 .

This behaviour is further illustrated in figure 14, where the relative angular position of the harvester is plotted for $l_{1}=10 \mathrm{~cm}$. Initially this angle rises rapidly, since the rotor is rotating with the source while the stator undergoes only small oscillations. At some speed, the stator flips over several times, but then stops, and this happens several times. Eventually, the motion of the stator is almost synchronized with the rotor, so that only small, and decreasing, oscillations in the relative angle are seen, corresponding to a decline in power. For the smaller offset $l_{1}=2 \mathrm{~cm}$, the stator remains held in place by gravitational torque, so that the generated power continues to rise. In general, for large offset distances, we can expect a maximum rotational speed beyond which the achievable output power drops rapidly.

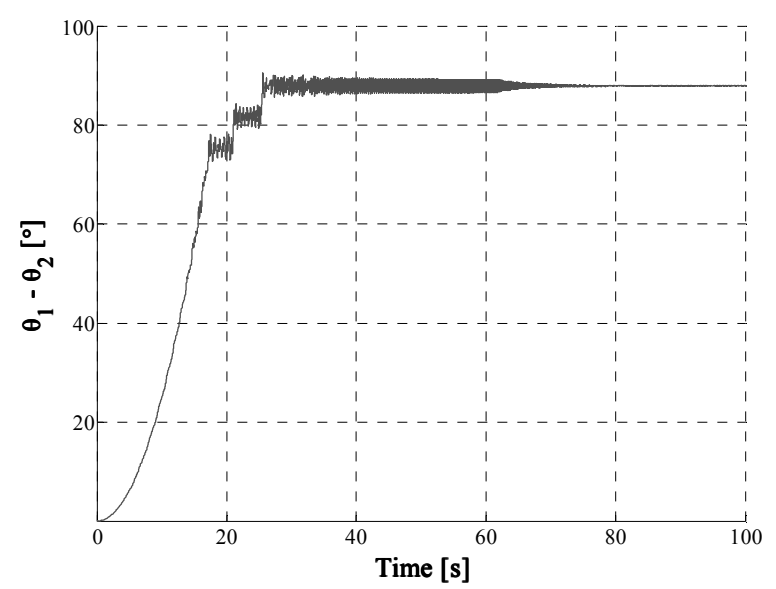

Figure 14. Relative angle, $\left(\theta_{l}-\theta_{2}\right)$ for $l_{l}=10 \mathrm{~cm}$.

\section{Scaling considerations}

In our experiments to date we have used $\mathrm{cm}$-scale, commercial DC motors for mechanical-to-electrical power conversion. These devices can deliver more power than would be required for many sensor applications, and are also relatively bulky (volume approximately $23 \mathrm{~cm}^{3}$ ). It is therefore of interest to consider how the rotational energy harvesting generator presented in this paper might be miniaturized, for example through the use of MEMS technology.

From basic scaling considerations it can be shown that the constant $K_{E}$ of a permanent magnet (PM) generator scales as $L^{2}$ where $L$ is a characteristic length [8]. This assumes that the device geometry and PM magnetization remain constant as the device size is changed. Under the same conditions, the armature resistance $R_{A}$ scales as $L^{-1}$. From these scaling laws it follows that the available output power, as given by (2), will scale as $L^{5}$ at constant rotational speed, or as $v o l^{5 / 3}$ where $v o l$ is the device volume.

The rapid scaling of the output power with size precludes significant down-sizing of PM generators. However, it is reasonable to expect that optimized mmscale generators should be capable of delivering useful levels of power at modest rotation speeds, and this potential has already been demonstrated by several groups [9]. For example, a team at Laboratoire d'Electronique de Grenoble (LEG) has recently reported a MEMS axial-flux PM generator with an active volume of only $23 \mathrm{~mm}^{3}$ that would be expected to deliver around $850 \mu \mathrm{W}$ if operated at $5000 \mathrm{rpm}$ [10].

Figure 15 shows one possible implementation of a rotational energy harvester based on an axial-flux PM generator. The PM generator in this example is similar 
in construction to one demonstrated previously by us [8]. It comprises a rotor with embedded permanent magnets sandwiched between two silicon stators carrying electroplated copper coils. An eccentrically mounted mass has been added to the rotor to allow operation as a rotational energy harvesting generator.

Successful realization of an efficient MEMS-based rotational energy harvester along the lines of figure 15 would require the implementation of suitable low-loss bearings. Also, the power conditioning electronics would need to be designed for efficient operation at low generator output voltages. The scaling of $K_{E}$ is such that mm-scale PM generators commonly have voltages in the $\mathrm{mV}$ range at modest rotational speeds. For example, the device reported by us in [8] had a $K_{E}$ of $1.34 \times 10^{-4}$ $\mathrm{V} \cdot \mathrm{s} / \mathrm{rad}$, corresponding to an output voltage of $70 \mathrm{mV}$ rms at $5000 \mathrm{rpm}$.

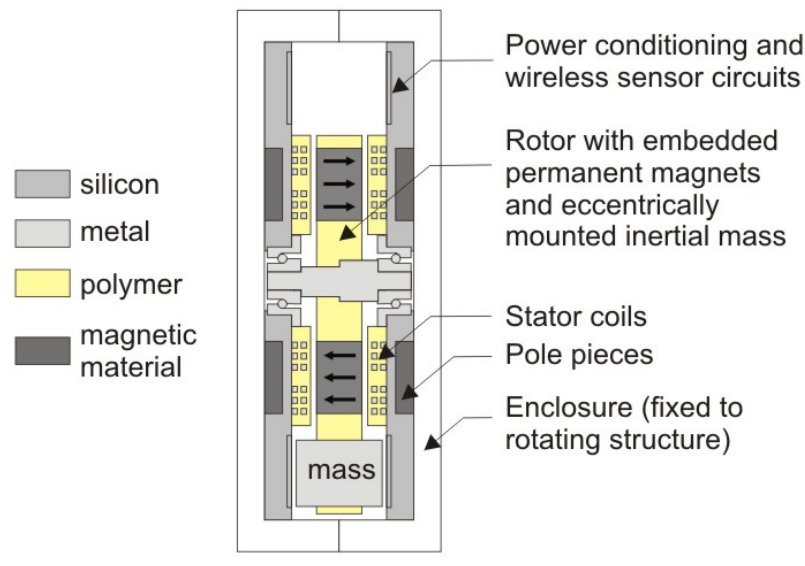

Figure 15. Possible MEMS implementation of microgenerator with integrated electronics.

\section{Conclusions}

The use of conventional DC machines for energy harvesting from continuous rotation has been demonstrated. The use of gravitational torque substitutes for the inertial effect which is typically used in energy harvesters, but cannot be used with continuous motion. We have also designed and demonstrated an adaptive power conditioning circuit, which implements maximum power point tracking. Finally, the use of the device when mounted off axis is evaluated, and shown to be possible within a limited range of source speed.

\section{References}

[1] J. A. Paradiso and T. Starner, Energy Scavenging for Mobile and Wireless Electronics, Pervasive Computing, IEEE, vol. 4, pp. 18-27, 2005.

[2] P. D. Mitcheson, T. C. Green, E. M. Yeatman, A. S. Holmes, Architectures for Vibration-Driven Micropower Generators, J. Microelectromechanical Systems, vol. 13, pp. 429-440, 2004.

[3] E.M. Yeatman, Energy Harvesting from Motion Using Rotating and Gyroscopic Proof Masses, J. Mechanical Engineering Science 222 (C1), pp. 27-36 (2008).

[4] M. Hayakawa, Electric Wristwatch with Generator, U.S. Patent 5,001,685, March, 1991.

[5] Tzern T. Toh, Paul D. Mitcheson, Eric M. Yeatman, A Gravitational Torque MicroGenerator For Self-Powered Sensing, in Micro Mechanics Europe '07, Guimarães, September 16-18, 2007, pp. 341-344.

[6] Microchip, http://www.microchip.com.

[7] Recom International Power, http://www.recominternational.com.

[8] A. S. Holmes, G. Hong, K. R. Pullen, Axial-Flux, Permanent Magnet Machines for Micropower Generation, J. Microelectromechanical. Systems, vol. 14, pp. 54-62, 2005.

[9] D.P. Arnold, Review of microscale magnetic power generation, IEEE Trans. Mag., vol. 43, no. 11, pp. 3940-3951, 2007.

[10] H. Raisigel, O. Cugat, J. Delamare, Permanent magnet planar micro-generators, Sens. Act. A, vol. 130-131, pp. 438-444, 2006. 